\title{
Coregulation of anterior and posterior mesendodermal development by a hairy-related transcriptional repressor
}

\author{
Laure Bally-Cuif, ${ }^{1,3,5,6}$ Carole Goutel, ${ }^{2}$ Marion Wassef,, ${ }^{1}$ Wolfgang Wurst, ${ }^{3,4}$ and Frédéric Rosa ${ }^{2,6}$ \\ ${ }^{1}$ Centre National de la Recherche Scientifique URA 1414, ${ }^{2}$ INSERM U368, Ecole Normale Supérieure, 75005 Paris France; \\ ${ }^{3}$ GSF-Forschungszentrum, Institut für Säugetiergenetik, D85764 Neuherberg Germany; ${ }^{4}$ Max-Planck-Institut für Psychiatrie, \\ D80809 München Germany
}

\begin{abstract}
During embryonic development in vertebrates, the endoderm becomes patterned along the anteroposterior axis to produce distinct derivatives. How this regulation is controlled is not well understood. We report that the zebrafish hairy/enhancer of split [E(spl)]-related gene her5 plays a critical role in this process. At gastrulation, following endoderm induction and further cell interaction processes including a local release of Notch/Delta signaling, her5 expression is progressively excluded from the presumptive anterior- and posteriormost mesendodermal territories to become restricted to an adjacent subpopulation of dorsal endodermal precursors. Ectopic misexpressions of wild-type and mutant forms of her5 reveal that her 5 functions primarily within the endodermal/endmost mesendodermal germ layer to inhibit cell participation to the endmost-fated mesendoderm. In this process, her5 acts as an active transcriptional repressor. These features are strikingly reminiscent of the function of Drosophila Hairy/E/spl) factors in cell fate decisions. Our results provide the first model for vertebrate endoderm patterning where an early regulatory step at gastrulation, mediated by her 5 controls cell contribution jointly to the anterior- and posteriormost mesendodermal regions.
\end{abstract}

[Key Words: Endoderm regionalization; zebrafish; her5; anterior and posterior mesendoderm; prechordal plate]

Received April 4, 2000; revised version accepted April 19, 2000.

Understanding how the germ layers of vertebrate embryos are formed and patterned is a fundamental issue in developmental biology; however, at present only the induction and regionalization of the ectoderm and mesoderm have been worked out in relative depth. The endoderm has been comparatively less approached. Recently, however, mutant mice and zebrafish affected in endoderm formation, and the cloning of Xenopus and zebrafish endoderm-specific genes, have opened new avenues to the understanding of endoderm development, and a first molecular pathway of endoderm induction was assembled (Alexander and Stainier 1999; Yasuo and Lemaire 1999; and references therein). The factors identified are expressed following endoderm induction, but none of them is present in a region-specific manner, suggesting that they are involved in maintaining (or inducing) a general endodermal specification, rather than imparting a regional character to their expressing cells.

${ }^{5}$ Present address: GSF-Forschungszentrum, Institut für Säugetiergenetik, D85764 Neuherberg Germany.

${ }^{6}$ Corresponding authors.

E-MAIL bally@gsf.de; FAX (49-89) 3187-3099.

E-MAIL rosa@wotan.ens.fr; FAX (33-1) 44-32-3988.
Similarly, mutations affecting the zebrafish endoderm at early stages impair endoderm formation as a whole, rather than its regionalization (Feldman et al. 1998; Alexander and Stainier 1999; Gritsman et al. 1999). Thus, a major open question now remains to identify the mechanisms and factors involved in the regionalization of endoderm, a most important issue given the capacities of specific endodermal subdomains or derivatives to organize other territories like the head, the heart, and the anterior CNS (Foley et al. 1997; Pera and Kessel 1997; Schneider and Mercola 1999).

In the zebrafish (Danio rerio), fate mapping studies have demonstrated that endodermal precursors are topographically arranged at the blastoderm margin at the onset of gastrulation, such that dorsally located cells tend to contribute to anterior derivatives (pharynx), whereas ventrolateral cells preferentially populate the posterior endoderm (gut) (Warga and Nüsslein-Volhard 1999) (Fig.1a). Endoderm regionalization might be expected to begin before or around the onset of gastrulation, establishing a prepattern along the blastoderm margin of the early gastrula. Marginal cells, however, can be repatterned to produce a complete set of endodermal derivatives (Peyrieras et al. 1998). This suggests that local cues 
a
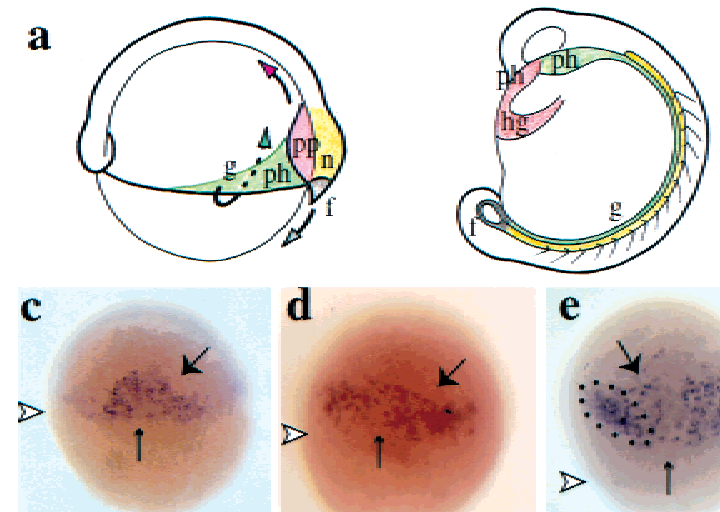

d $\quad$ e
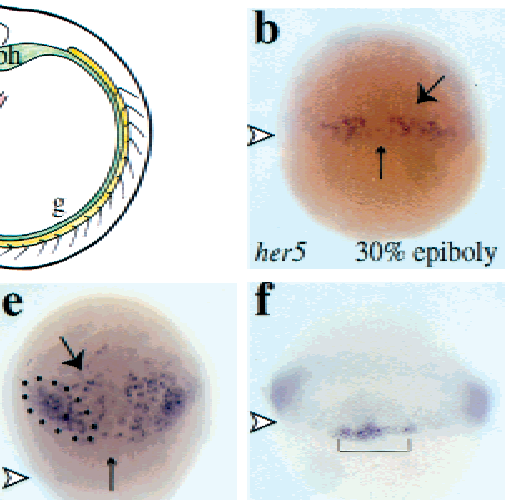

f

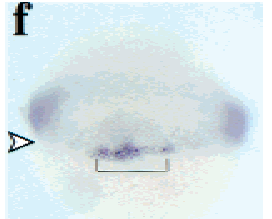

her5 shield her5 $60 \%$ epiboly her 5

$70 \%$ epiboly $s q t$
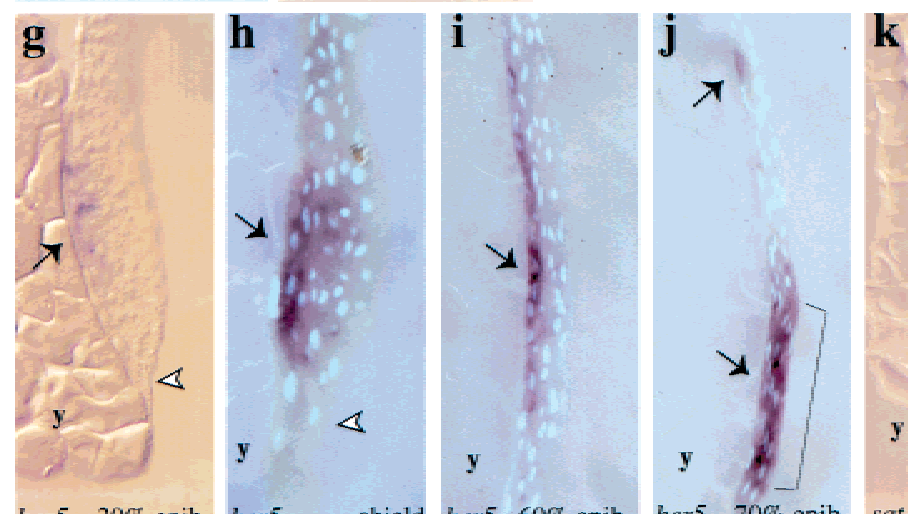

shield
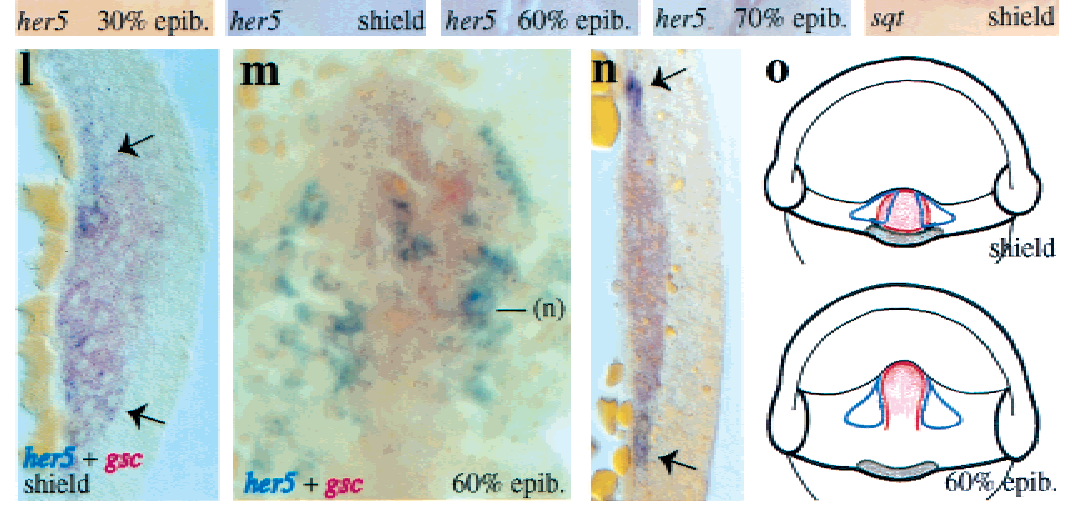

Figure 1. her5 is expressed in a subset of presumptive endodermal/mesendodermal cells at gastrulation. (a) Simplified fate map of the endoderm and mesendoderm at early gastrulation (from Shih and Fraser 1995; Cooper and D'Amico 1996; Melby et al. 1996; Warga and Nüsslein-Volhard 1999). The presumptive territories at the shield stage (left) and their derivatives at the 15somite stage (right) (lateral views, dorsal to the right) are color-coded, and cell movements are indicated by arrows. her 5 and sqt expression revealed by in situ hybridization (blue) in wholemount gastrulae $(b-f)$ and on the corresponding parasagittal sections $(g-k)$ at the stages indicated (lower right corner), anterior to the top (white arrowheads point to the blastoderm margin). From 30\% epiboly on, her5 (large arrows on $b-e, g-j)$ is expressed in a subpopulation of dorsal deep cells (the small arrow indicates the dorsal midline), it becomes restricted to dorsal endodermal precursors immediately following the shield stage. her5-positive cells are located in direct apposition to the yolk syncitial layer $(g-j)$. From $70 \%$ epiboly, her5 expression is also turned on in the presumptive mid-hindbrain (dots in $e$, bracket in j). At that stage, her5-positive endodermal precursors are restricted to the prechordal embryonic area. At the shield stage, sqt expression selectively labels forerunner cells (brackets in $f$, and the her5- and sqt-positive domains are adjacent and nonoverlapping (cf. $c$ and $f, h$ and $k$ ). her5 (blue) and gsc (red) expression at shield (1) and $60 \%$ epiboly $(m, n)$ stages $(m$, dorsal view, anterior to the top; $1, n$, sections of the prechordal plate, dorsal to the right; contrary to sections $g-k, 1$ and $n$ are cross-sections, oriented vertically). her5 (large arrows) and gsc expression overlap in the lateral aspects of the organizer at the shield stage, but are exclusive by $60 \%$ epiboly. In $m$, the medial her5-expressing cells are located under the gsc-positive prechordal plate. (o) Schematic of the spatial relationship of her5 (open blue domains), gsc (red), and sqt (brown) expression patterns at the shield (top) and $60 \%$ epiboly (bottom) stages (dorsal views).(f) forerunner cells and derivatives; $(\mathrm{g})$ gut precursors; (hg) hatching gland; (n) notochord; (ph) pharyngeal precursors; (pp) prechordal plate; (y) yolk. control and restrict the acquisition of specific endodermal identities during the course of normal development. Regionalization of the endodermal germ layer might depend partly on the dorsal mesoderm specification pathway (Henry et al. 1996; Joseph and Melton 1998; Yasuo and Lemaire 1999; Zorn et al. 1999). However, the factors responding to these positional cues remain entirely unknown.

We have searched for region-specific markers of the presumptive endoderm at gastrulation in the zebrafish and found that the gene her5 (Müller et al. 1996) was specifically expressed in a dorsal subpopulation of endodermal precursors. her5 was initially isolated in a PCR screen against zebrafish hairy/enhancer of split
E(spl) family members (v. Weisäcker 1994). Hairy/E(spl) factors are basic helix-loop-helix region (bHLH) transcription factors acting as transcriptional repressors, and differ from other bHLH factors by a proline residue in its basic DNA-binding domain, and by a carboxyterminal WRPW amino acid motif. In both Drosophila and vertebrates, these features have been proposed to confer unconventional DNA-binding specificity to bHLH proteins and to permit the recruitment of Groucho-like cotranscriptional repressors, respectively (Fischer and Caudy 1998, and references therein). Hairy/ E(spl) proteins mediate cell fate specification choices during multiple developmental processes, including neurogenesis and myogenesis, where $\mathrm{E}(\mathrm{spl})$ factors are 
direct transcriptional targets of Delta/Notch signaling (Fischer and Caudy 1998). The vertebrate-related proteins HES-1, HES-5, and her4 seem to play very similar roles during neurogenesis (Lee 1997; Fischer and Caudy 1998; Takke et al. 1999|, whereas her1 and c-hairy-1 (Palmeirim et al. 1997; Takke and Campos-Ortega 1999) contribute to the segmentation of the paraxial mesoderm. Zebrafish her5 was not studied functionally, but was noticed for its specific expression in the presumptive midbrain in late gastrulae, suggestive of a role in defining the midbrain anlage (Müller et al. 1996). Here we show that an earlier phase of her5 expression at gastrulation is restricted to a subdomain of the endoderm that becomes adjacent to the dorsal cell populations fated to the anterior- and posteriormost mesendoderm (we define the mesendoderm as the deep organizer-derived territories that cannot be unambiguously assigned to endoderm or mesoderm, i.e., the prechordal plate and forerunner cell population, see below). In addition, we demonstrate that her5 is able to specifically control the number of cells allocated to these endmost-fated mesendodermal populations. Thus, our results identify the first regionalized, endoderm-specific factor in vertebrates, and illustrate its pivotal role in patterning the deep embryonic layers in the zebrafish gastrula. In addition, we analyzed some of the cellular and molecular processes involved in regulating her5 expression within the endodermal germ layer. Taken together, our results allow for the building of a model for the regionalization of the (mes)endodermal layer in the vertebrate embryo.

\section{Results}

her5 is progressively restricted to a subset of dorsal endodermal precursor cells at gastrulation

Systematic in situ hybridizations in blastula-stage zebrafish embryos revealed an initial ubiquitous expression of her5 at the onset of zygotic transcription (data not shown), which rapidly resumed in a first, transient, and spatially restricted phase of her5 expression within the presumptive endoderm and mesendoderm (Fig.1). At $30 \%$ epiboly, her5 is selectively expressed in deep, scattered cells of the dorsal embryonic margin (Fig.1b,g). In the early gastrula (shield stage; Fig.1c,h), her5-positive cells remain scattered and confined to the deepest layer of the dorsal organizer, and from midgastrulation onwards (60\% epiboly stage), they are localized in the deep and anterior component of the dorsal hypoblast, in direct apposition to the yolk syncitial layer (Fig.1e,i). From that stage, the deep and scattered distribution of her5-positive cells, their flattened appearance (data not shown), together with their coexpression of the general endodermal markers axial (Strähle et al. 1993) and gata5 (Rodaway et al. 1999; data not shown), strongly suggest that her5 expression is restricted to a subpopulation of endodermal precursors (Warga and Nüsslein-Volhard 1999; below). At the end of gastrulation, comparisons with landmarks of the prechordal/epichordal junction, such as the anterior limit of the notochord (data not shown) or the mid-hindbrain (Fig.1e,j), indicate that the her5-positive cells are confined to the prechordal embryonic area. Expression of her5 in the presumptive endoderm is then progressively extinguished.

In the zebrafish, specification of the endoderm/mesendoderm germ layer requires the diffusible Nodal signals cyclops (cyc) and squint (sqt), as well as one-eyed-pinhead (oep), a membrane bound protein essential for Nodal signaling and acting downstream of $c y c$ and sqt (Schier and Shen 2000). In addition, an activated form of the TGF $\beta$-type I receptor Taram-A $\left(\operatorname{Tar}^{\star}\right)$ triggers the formation of endoderm and endmost mesendoderm, and acts downstream of Nodal signals (Peyrieras et al. 1998). We observed that her 5 was never expressed in gastrulating oep embryos, and in contrast, was strongly induced upon misexpressions of $\operatorname{Tar}^{\star}$ (data not shown). her5 induction was then rapidly regulated during gastrulation to be maintained in a limited number of Tar ${ }^{\star}$-expressing cells only (data not shown). Thus, the expression of her5 at gastrulation lies downstream of the endoderm $/$ mesendoderm specification cascade, further supporting the notion that it is restricted to this germ layer.

Taken together, our results indicate that her5 is expressed in a subpopulation of dorsal, anterior-fated endodermal/mesendodermal precursors at gastrulation, and thus constitutes the earliest known regional marker of the presumptive endoderm.

At early gastrulation stages, her5 expression is progressively excluded from the endmost-fated mesendodermal territories

In Drosophila, Hairy-related bHLH factors are involved in delimiting expression territories and/or domains of cell specification within the embryo and larva (Fisher and Caudy 1998). To get a hint regarding which genes or territories might be influenced by her 5 expression during the course of gastrulation, we compared precisely the location of her5-expressing cells with identified subdomains of the dorsal embryonic margin.

From the onset of gastrulation, her5-positive endodermal precursors showed a tendency to distribute away from the dorsal midline (Fig.1b-e). We compared her5 expression with the dorsomedial mesendodermal marker goosecoid (gsc) (Stachel et al. 1993; Schulte-Merker et al. 1994; Thisse et al. 1994). At the shield stage, the medial area of the organizer expresses exclusively gsc, while its lateral aspects (5-6 cell rows on either side) coexpress the two genes (Fig. 11). More laterally, only her5 expression is found. Importantly, the gsc and her5 expression territories rapidly become exclusive. By the $60 \%$ epiboly stage, the gsc-positive prechordal plate is flanked by 1-2 rows of her5-expressing cells; cells expressing both transcripts are no longer observed (Fig. 1m,n). This relative distribution is maintained until her 5 expression is extinguished at the end of gastrulation (not shown). Later, as shown in Figure 1a, cells of the gsc-positive territory will contribute to the anteriormost mesendodermal derivatives, including the hatching gland, head mesoderm, and a limited part of the pharynx (Shih and 
Fraser 1995; Melby et al. 1996; Warga and Nüsslein-Volhard 1999).

From the onset of gastrulation, her5-expressing cells are found near the edge of the dorsal blastoderm margin (Fig. 1b,c,g,h). We compared the location of her5-positive cells with a group of dorsal marginal cells known as forerunners [Fig. If (bracket),1k]. The forerunner cluster is located at the leading edge of the advancing blastoderm margin (Cooper and D'Amico 1996); from the shield stage, it expresses selective molecular markers, including the gene sqt (Feldman et al. 1998; Rebagliati et al. 1998). In shield-stage embryos, the her5- and sqt-positive territories are adjacent and nonoverlapping (Fig. 1c,f,h,k), thus strongly suggesting that her 5 is expressed in cells abutting the forerunner cells cluster. As forerunner cells do not involute during gastrulation but migrate toward the vegetal pole, the two domains rapidly segregate from each other. Later, the forerunner cells will contribute to the posteriormost mesendodermal derivatives, expressing mesendodermal fates in the tail at the base of the notochord and postanal gut endoderm (Fig. 1a), and later in notochord tip cells and tail mesenchyme (Cooper and D'Amico 1996; Melby et al. 1996).

Thus, her5 expression is initially contiguous or overlapping with, but is progressively excluded from, two subdomains of the dorsal mesendoderm expressing the markers gsc and sqt (Fig. 1o), and fated to the anteriorand posteriormost mesendoderm, respectively.

\section{her5 expression controls the number of cells expressing goosecoid at gastrulation and acquiring anterior mesendodermal fate}

The above observations, together with the function of the her5-related Drosophila factors, suggested that her5 expression might influence the limits of the gsc-and $s q t$-positive domains at gastrulation, and the formation of the corresponding anterior and posterior mesendodermal derivatives. To test this hypothesis, we misexpressed wild-type and mutant forms of her5 (Fig. 2a) in zebrafish embryos. Injections of her5 RNA at the onecell stage were used to study the consequences of her5 misexpressions during gastrulation.

The Hairy/E(spl)-related factors studied to date act as transcriptional inhibitors. However, their molecular modes of action vary depending on the developmental context (Fischer and Caudy 1998). For example, whereas Hairy/E(spl) proteins generally behave as active transcriptional repressors (i.e., require DNA-binding at specific target sites), cases of transcriptional repression in-

a
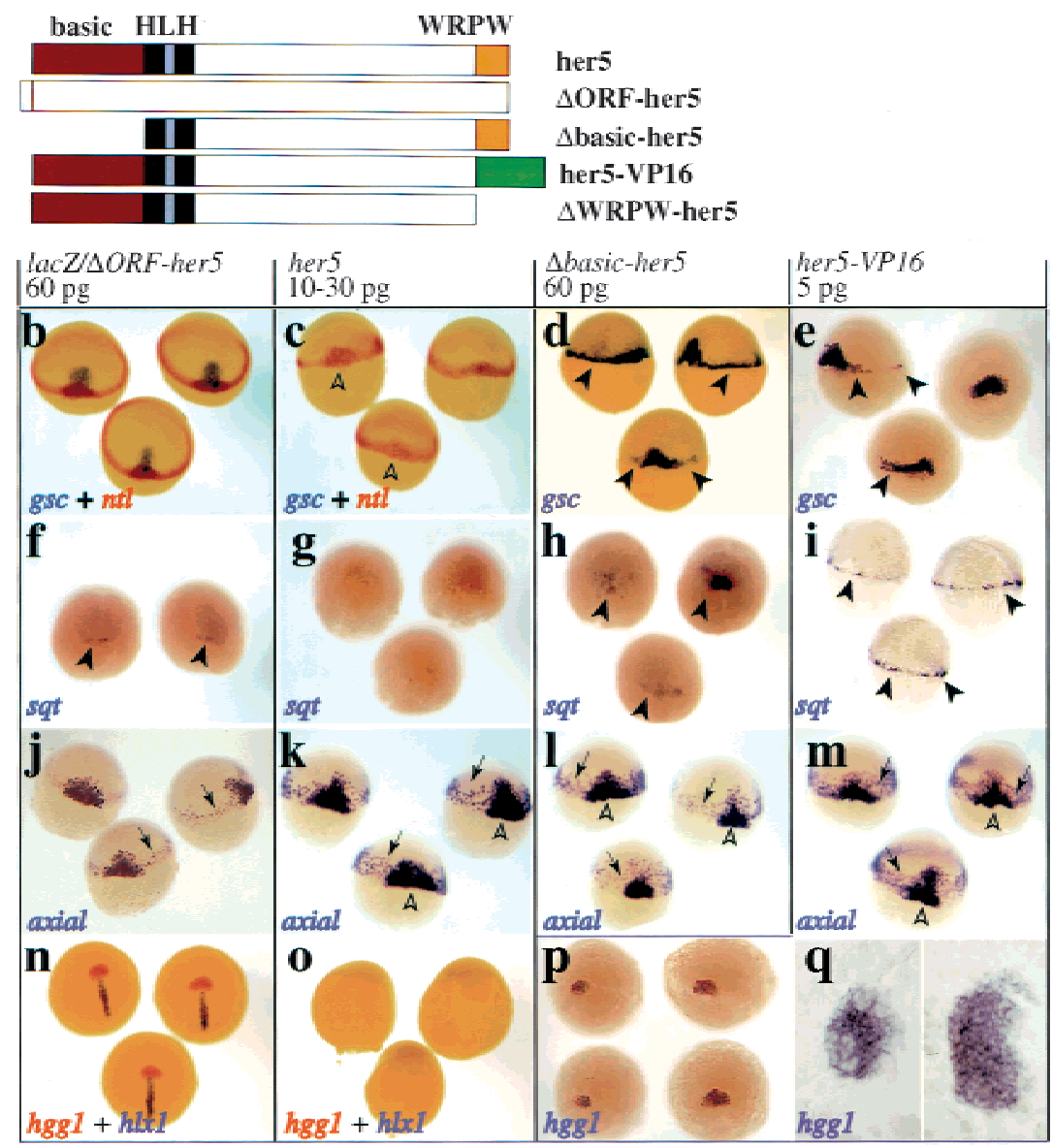

Figure 2. her5 controls the number of cells specified to the anterior- and posterior-most mesendoderm during gastrulation. (a) Wildtype and mutant forms of her5 (red; basic DNA-binding domain; black, HLH dimerization domain; yellow, WRPW carboxy-terminal tetrapeptide; green, VP16 activation domain). $(b-q)$ Embryos were injected at the one-cell stage with capped RNAs as indicated above each boxed area, and probed at shield- $60 \%$ epiboly $(b-m)$ or $90 \%$ epiboly $(n-q)$ with the genes indicated on each panel (color-coded). Dorsal views, anterior to the top, except in $m$ (side views; d, dorsal side) and $q$ (flat-mounts of the embryos in $p$, anterior to the left). her5 misexpressions inhibit gsc, sqt, and hgg1 expression $(c, g, O)$. In $n-O, h l \times 1$ labels the posterior part of the prechordal plate, so most of the prechordal plate is missing in her5-injected embryos. Conversely, $\Delta$ basic-her5 and her5VP16 increase the number of cells expressing gsc and sqt (d,e,h,i, arrowheads). The effect of her5-VP16 perdures and induces 2.5 times more hgg1-positive cells at late gastrulation $(p, q$; cf. control and her5-VP16-injected embryos on the left and right of each panel, respectively). The endoderm fated to intermediate anteroposterior positions ( $k-m$, arrows) is not significantly affected; neither are notochordal precursors [medial expression of $n t l(c)$ and axial $(k-m)$ ] (open arrowheads; data not shown). 
dependent of DNA-binding, or by direct interference with transcriptional activators, have been reported in Drosophila (Fischer and Caudy 1998). Furthermore, in some instances, interactions with Groucho-like cotranscriptional repressors appear dispensable (Dawson et al. 1995; Takke and Campos-Ortega 1999; Takke et al. 1999). We designed variants of her5 to test these different functional requirements (Fig. 2a). $\Delta$ basic-her5 lacks the basic DNA-binding domain of her5, but leaves the helixloop-helix dimerization domain intact. $\Delta$ WRPW-her5 lacks the WRPW Groucho interaction site. In her5VP16, the WRPW tetrapeptide was deleted and replaced by two copies of the minimal activation domain of VP16 (Seipel et al. 1994). Finally, $\Delta$ ORF-her5 carries a premature Stop codon in a Myc-her5 fusion construct, resulting in the production of a truncated protein including the Myc epitope but not the her5 protein. Capped $\triangle O R F-$ her5 or lacZ mRNAs were used indiscriminately as negative controls in all experiments reported below, without ever causing detectable embryonic abnormalities.

Ectopic expression of wild-type her5 from the one-cell stage dramatically decreased the number of cells expressing gsc at the onset of gastrulation $190 \%$ decrease in $80 \%$ of cases, $n=87$ ) (Fig. 2b,c). On the contrary, expression of $\Delta$ basic-her5 and her5-VP16 increased the number of gsc-expressing cells at the blastoderm margin $1>2$-fold in $>50 \%$ of cases, $n>25$ ) ( Fig. 2 d,e). In addition, $\Delta$ basic-her 5 and her5-VP16 behaved as dominant-negative forms of her 5 because they blocked its effects in coinjection experiments (in $>88 \%$ of cases, $n>15$ ) (data not shown). The dominant-negative effect of $\Delta$ basic-her5 is consistent with previous studies of other bHLH factors (Fischer and Caudy 1998) and suggests that $\Delta$ basic-her5 acts via the formation of nonfunctional dimers with the endogenous her5 protein. Thus her5 activity likely requires both DNA-binding and dimerization. The dominant-negative effect of her5-VP16 can be best explained if her5-VP16 retains the DNA-binding and dimerization specificities of her5, but is turned into a transcriptional activator, as demonstrated for other inhibitory proteins including Hairy itself (Jimenez et al. 1996; Ermakova et al. 1999; Zuber et al. 1999). Because her5 is the only her-family member expressed at such an early stage of zebrafish development (v. Weiszäcker 1994), it is likely that $\Delta$ basic-her5 and her5-VP16 selectively interfere with endogenous her5 in our injection experiments. Finally, $\Delta$ WRPW-her5 was inactive on gsc expression, as well as at the later stages studied below (data not shown), indicating that the WRPW motif (and probably Groucho-like factors) is required for her 5 activity. Thus, our results strongly suggest that, at gastrulation, her5 acts as an active inhibitor of transcription to control the number of cells expressing gsc at the gastrula margin.

Cells expressing gsc during gastrulation normally contribute to the anteriormost mesendoderm, including the hatching gland. Accordingly, her5 expression regulated the formation of anterior mesendodermal derivatives at late stages. At the end of gastrulation, her5 misexpres- sion blocked expression of the hatching gland marker hgg1 (Thisse et al. 1994) (85\% of cases, $n=36$ ) ( Fig. 2o), while her5-VP16 markedly increased the number of hgg1-positive cells (2.5-fold in 50\% of cases, $n=12$ ) (Fig. $2 \mathrm{p}, \mathrm{q})$.

To analyze the effects of her5-variants at later stages, injections were carried out into one marginal blastomere at the 16-cell stage, together with a fluorescent tracer. Such injections allowed us to trace potential lineage alterations in the progeny of the injected blastomere. Embryos injected dorsally with her5 (Fig. 3) never developed a hatching gland $(n=48)$ and exhibited partial or complete cyclopia (65\% and $35 \%$ of cases, respectively) (Fig. $3 \mathrm{a}, \mathrm{b})$. Those defects in all cases correlated with a mispatterning of the ventral forebrain (data not shown). Importantly, none of the studied embryos $(n=48)$ displayed abnormal heart or pharyngeal structures, or abnormal gut and notochord (see Fig. 3d,f, and below). In spite of their effects at late gastrulation (Fig. 2p,q), $\Delta$ basic-her5 and her5-VP16 injections at any dorsoventral location did not lead to the formation of significantly larger or ectopic hatching glands at these stages $\mid n>100$, data not shown), possibly due to embryonic regulation. Taken together, these results indicate that her5 expression at gastrulation controls the number of cells specified to contribute to the anteriormost mesendoderm, and the subsequent development of anterior mesendodermal derivatives. In this process, her5 functions probably as an active transcriptional inhibitor, and requires dimerization and its carboxy-terminal WRPW tetrapeptide, known to interact with Groucho factors in structurally related molecules.

her5 expression controls the number of cells expressing sqt at gastrulation and acquiring posterior mesendodermal fate

her5 had a similar effect on sqt expression in forerunner cells and on the subsequent formation of the posteriormost mesendoderm. Misexpression of wild-type her5 dramatically decreased the number of cells expressing sqt at the shield stage (5-fold decrease in $>90 \%$ of cases, $n=32$ ) (Fig. 2f,g). In contrast, $\Delta$ basic-her5 and her5VP16 increased the number of sqt-expressing cells (2.5and 5 -fold increase, respectively, in $>65 \%$ of cases, $n>20$ ) (Fig. 2h,i). The ectopic sqt-positive cells induced by $\Delta$ basic-her5 were confined to the dorsal embryonic area, whereas those following her5-VP16 expression were distributed around most of the blastoderm margin (Fig. 2, cf. $\mathrm{h}$ with i). Other forerunner cell markers [such as no tail (ntl) and sox17] (Schulte-Merker et al. 1992; Alexander and Stainier 1999) confirmed these findings (data not shown) and suggested that the regulation of sqt expression matched the regulation of forerunner cells. Again, in this assay, $\triangle \mathrm{WRPW}-$ her5 was inactive (data not shown), while both $\Delta$ basic-her5 and her5-VP16 behaved as dominant-negatives. The different effects of the two latter constructs on $s q t$ expression further indicate that her5-VP16, in contrast to $\Delta$ basic-her5, does not behave 

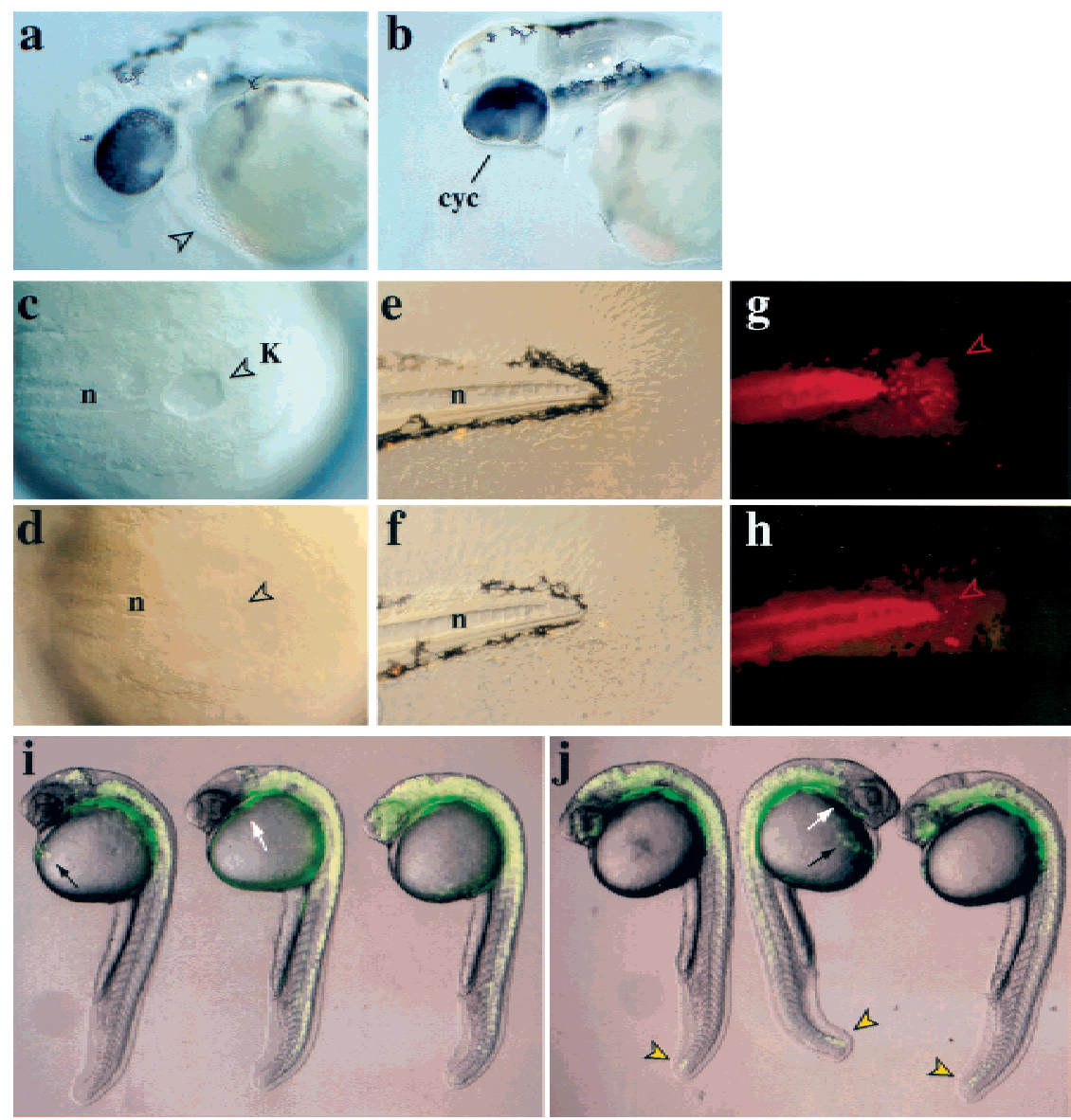

Figure 3. her5 misexpression impairs the formation of the anterior- and posteriormost mesendodermal derivatives. Embryos were injected into a dorsal marginal blastomere at the 16-cell stage with $6 \mathrm{pg}$ of lac $Z(a, c, e)$ or 6 pg of $1 a c Z+1-3$ pg of her5 RNAs $(b, d, f)$ and 2000S rhodamine dextran (anterior to the left). At $36 \mathrm{hr}$, her5-injected embryos lack hatching gland (arrowhead in a) and display synopthalmia (cyc) (cf. $a$ and b). Embryos were injected into a dorsal marginal blastomere at the 16-cell stage with 6 pg of $l a c Z(c, e, f)$ or 6 pg of $1 a c Z+1-3$ pg of her5 RNAs $(d, f, h)$ and $2000 S$ rhodamine dextran, and tail development was observed at 8 somites $(c, d)$ (bright field, dorsal views) or $48 \mathrm{hr}(e-h)$ (bright field and fluorescence views, lateral views; posterior to the right). Kupffer's vesicle ( $\mathrm{K}$, arrowheads) $(c, d)$ and forerunner derivatives in the tail fin mesenchyme ( $g, h$, arrowheads) are absent upon her5 expression. The notochord (n) is never affected (cf. $c$ and $d, e$ and $f$. Embryos were injected in a lateral marginal blastomere at the 16-cell stage with 5 pg of GFP (i) or 5 pg of GFP + $0.5 \mathrm{pg}$ of her5-VP16 (j) RNAs. Composite fluorescent and bright field images are shown. At $24 \mathrm{hr}$, her5-VP16 has induced the development of forerunner derivatives $(j$, arrowheads) from the lateral margin, which normally never contributes to this cell population (see i). Other endodermal domains (pharynx, white arrows; hatching gland, black arrows) are unaffected. (hg) hatching gland; (K) Kupffer's vesicle; (n) notochord. simply as an antimorphic form of her5, but has a positive transcriptional activity of its own. Thus, her5 acts as an active inhibitor of transcription to regulate the number of forerunner cells at gastrulation.

Consistent with the reported gastrulation defects, misexpression of her5 affected the formation of posterior mesendodermal derivatives at late stages (Fig. 3c-i). During somitogenesis, the forerunner cells are transiently incorporated into the epithelium lining Kupffer's vesicle, a teleost-specific structure continuous to the gut (Cooper and D'Amico 1996; Melby et al. 1996) (Fig. 3c). Half the embryos injected dorsally with her5 $(n=20)$ had either a reduced or absent Kupffer's vesicle (Fig. 3d). Accordingly, at two days, the number of forerunner-derived mesenchymal tail cells was strongly reduced (Fig. 3e-h). In contrast, misexpression of her5-VP16 in dorso-lateral or lateral regions of the margin induced forerunner cells to develop from these ectopic locations in $>85 \%$ of cases $(n=44)$ (Fig. 3i,j). These cells expressed sqt during gastrulation, participated to the epithelium of Kupffer's vesicle (data not shown) and later to the forerunner-characteristic fates in the tail (Fig. 3j).

Thus, her5 also functions as an active transcriptional inhibitor to control the number of cells specified to contribute to the posteriormost mesendoderm, and the sub- sequent development of posterior mesendodermal derivatives.

her5 expression does not affect mesendodermal precursors fated to intermediate anteroposterior levels

In contrast to the anterior- and posteriormost mesendoderm, perturbations of her5 expression did not affect mesodermal or endodermal precursors fated to intermediate anteroposterior locations. At gastrulation, markers of the presumptive intermediate endoderm such as axial (Fig. 2j-m) (n>70) or sox17 (data not shown; Strähle et al. 1993; Alexander and Stainier 1999|, or general markers of the blastoderm margin (see $n t l$, Fig. 2b,c) (SchulteMerker et al. 1992) or of the presumptive notochord [floating-head (flh); data not shown] were not significantly affected by the expression of wildtype or $\Delta$ basicher5. her5-VP16 generally increased the level of expression of endodermal markers (see axial, Fig. $2 \mathrm{~m}, n>40$; and sox17, not shown), but their expression profiles were maintained. At $24 \mathrm{hr}$, all her5-injected embryos expressed the pharyngeal marker $n k x 2.7$ (data not shown). Furthermore, when her5 was misexpressed in a dorso- or ventrolateral location along the blastoderm margin, the progeny of her5-expressing cells contributed normally to 
Figure 4. her5 misexpression does not affect intermediate endodermal derivatives. Embryos were injected into a dorsolateral $(a-c)$ or lateral $(d-f)$ blastomere at the 16cell stage with $4.5 \mathrm{pg}$ of her $5+5 \mathrm{pg}$ of GFP mRNAs, and are observed at $72 \mathrm{hr}$. $(a, b, d, e)$ Bright field and fluorescence whole-mount views, anterior to the left. $(c, f)$ Cross-sections at the levels indicated, with GFP protein revealed by immunocytochemistry (brown). The progeny of the injected blastomere (arrows) contributed normally to the pharyngeal pouches $(a-c)$ and gut derivatives $(d-f)$. $(f)$ Cell labeling in the swim bladder (area surrounded by filled dots; open dots line the intestine proper).
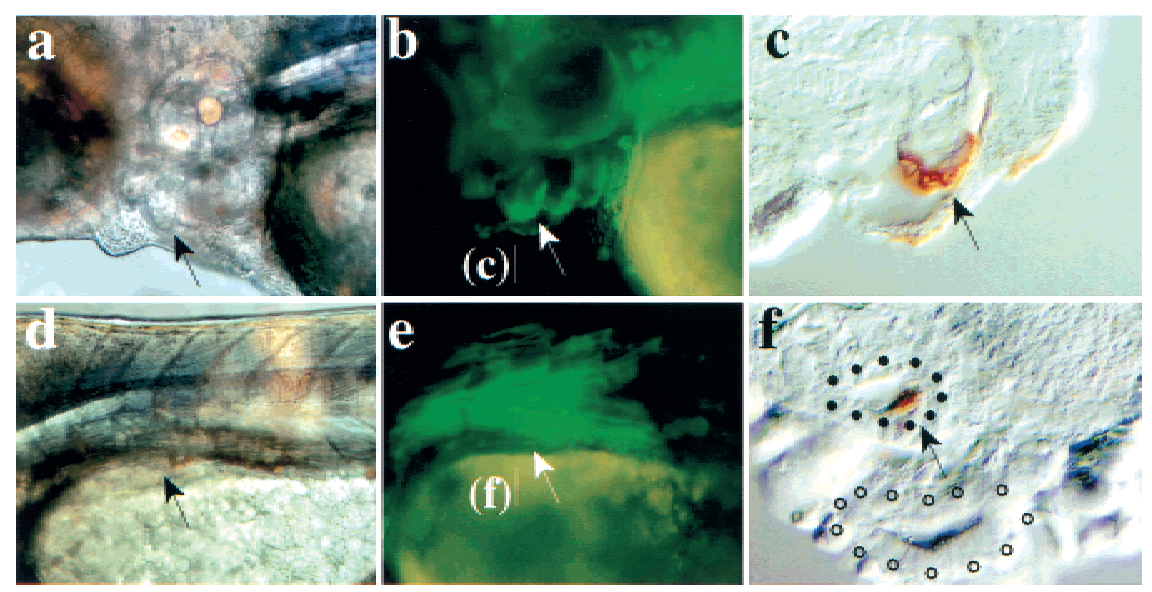

the epithelium of the pharyngeal pouches $163 \%$ of cases, $n=31$ ) (Fig. $4 \mathrm{a}-\mathrm{c}$ ) and to gut derivatives $(41 \%$ of cases, $n=66$ ) (Fig. 4d-f) at three days. Pharyngeal and gut development were equally unaffected when $\Delta$ basic-her5 (data not shown) and her5-VP16 (see Fig. 3n) were expressed along lateral aspects of the embryonic margin. Similarly, notochordal development appeared normal following the misexpression of all her5-related constructs on the dorsal embryonic side (Fig. $3 \mathrm{~h}, \mathrm{j}_{\text {; data not }}$ shown).

Taken together, our results therefore suggest that her5 expression at gastrulation selectively regulates the extent of the territories fated to the anterior- and posteriormost mesendodermal domains, without affecting mesendodermal derivatives of intermediate location.

her5 acts primarily within the endodermal/ mesendodermal lineage to control cell contribution to the endmost-fated mesendoderm

Endogenous her5 expression at gastrulation is normally restricted to endodermal and mesendodermal precursors. To meet this tissue specificity in our ectopic expression expriments, we made use of $\mathrm{Tar}^{\star}$, the expression of which drives all early blastomeres towards an endodermal-mesendodermal behavior and fate (Peyrieras et al. 1998). Irrespective of their location along the margin,
Tar*-expressing cells selectively contribute to the pharynx and to the gut, as well as to the anterior- and posteriormost mesendodermal domains, the hatching gland, and forerunner cells (Peyrieras et al. 1998) (Fig. 5a) (86\% of cases, $n=59$ ). We restricted her5 misexpression to these populations by coinjecting her5 and Tar* RNAs, together with a lineage tracer, into one blastomere at the 16-cell stage. In the presence of her5 transcripts, Tar* expressing cells were still directed to an endodermal/ mesendodermal fate (Fig. 5b) (100\% of cases, $n=79)$, and differentiated normally as assessed at three days (data not shown). However, they contributed to intermediate structures only (pharynx and gut), and not to the most anterior and posterior domains, the hatching gland, and tail bud (94\% of cases, $n=79$ ) (Fig. 5b). This phenomenon was not observed with her5 mutant forms (Fig. 5c; $74 \%$ of cases, $n=57$; and data not shown). From these observations, we conclude that her5 acts primarily within the cell population directed to the endodermal and endmost mesendodermal domains.

\section{her5 expression biases the specification choices of endodermal/mesendodermal progenitors}

We directly tested whether her5 expression affected cell specification choices within the presumptive endoder$\mathrm{mal} /$ mesendodermal population. Fate maps carried out

Figure 5. her5 misexpression in cells fated to the endoderm and endmost mesendoderm prevents their contribution to the endmost embryonic domains. Composite fluorescent and bright field views are shown, anterior to the left. $(a-c)$ 15-Somite stage. Embryos were injected into one marginal blastomere at the 16-cell stage with: $0.06 \mathrm{pg}$ of $\operatorname{Tar}^{*}(a), 0.06 \mathrm{pg}$ of $\operatorname{Tar}^{*}+3 \mathrm{pg}$ of her5 $(b), 0.06 \mathrm{pg}$ of $\mathrm{Tar}^{*}+3 \mathrm{pg}$ of $\Delta$ basicher5 RNAs $(c)$, and 2000S fluorescein dextran. A complete anteroposterior set of endodermal derivatives (green) is induced by
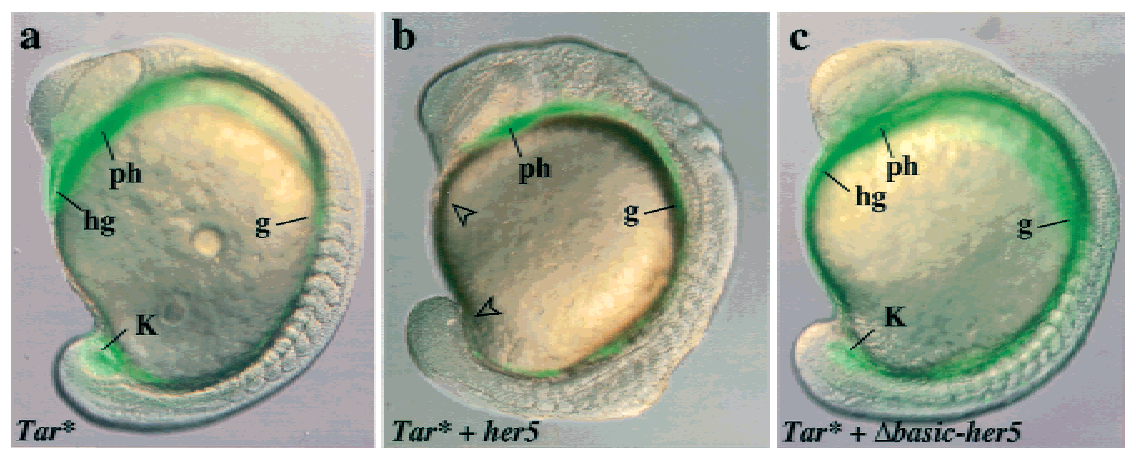
Tar $^{*}(a)$ and Tar* $+\Delta$ basic-her5 (c). (b) Tar* + her5-injected cells (green) contribute to intermediate endoderm [(ph) pharynx; (g) presumptive gut] but not the anteriormost [(hg) hatching gland] and posteriormost [(K) Kupffer's vesicle] domains (open arrowheads in $b$ ). 
in late blastulae embryos (40\% epiboly) and relying on the iontophoretic injection of the fluorescent tracer Fluorescein-Dextran (FD) have shown that the most marginal blastomeres (rows 1-4) located on the dorsal side give rise predominantly to hatching gland and pharyngeal endoderm cells, which often share common progenitors at this stage (Warga and Nüsslein-Volhard 1999|. We have carried out similar experiments by injecting the photoactivatable version of FD and uncaging the dye at the $40 \%$ epiboly stage in a single of the most marginal blastomeres by means of a microlaser beam (Serbedjiza et al. 1988) (Fig. 6a). Consistent with published results (Warga and Nüsslein-Volhard 1999), we find that most blastomeres labeled in such conditions
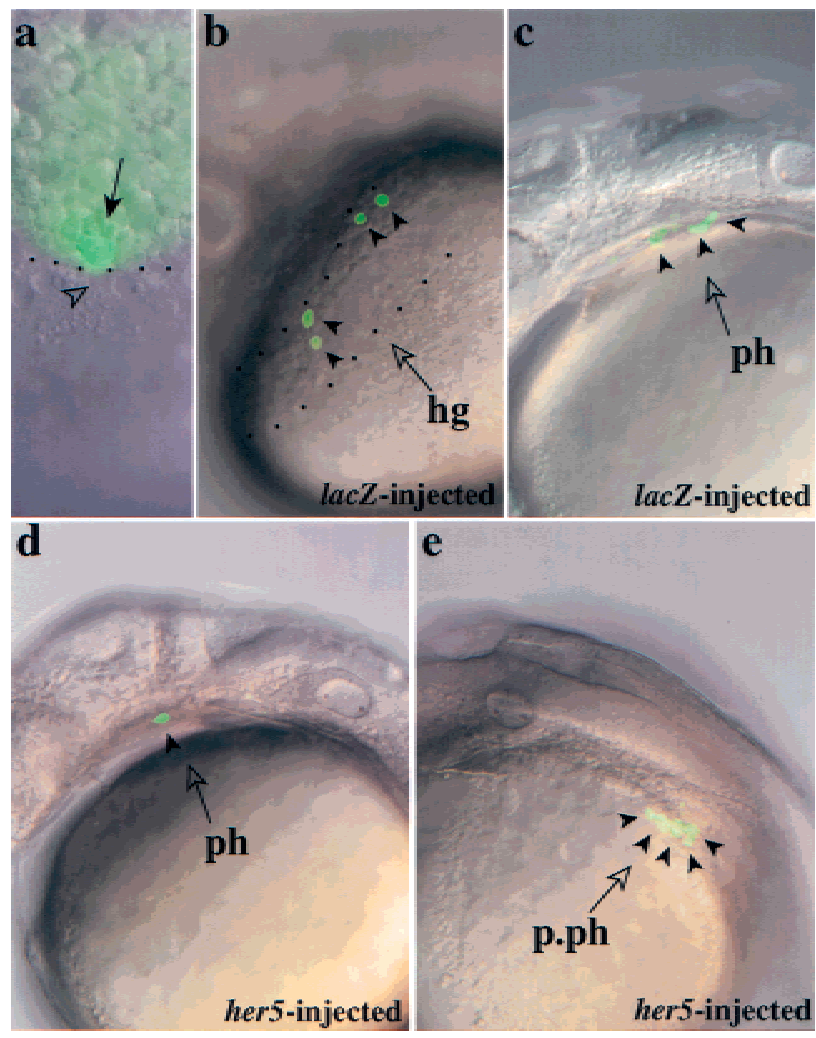

Figure 6. her5 expression biases cell fate choice within the endoderm/mesendoderm. (a) Fate mapping technique. Single dorsal blastomeres (arrow) of the most marginal row were labeled at $40 \%$ epiboly by uncaging photoactivatable Fluorescein DMNB with a microlaser beam (dots indicate the embryonic margin and the arrowhead points to a nucleus of the yolk syncitial layer). (b,c) Fate acquired by dorsal endomesendodermal precursors in wild-type embryos. Most progenitors contributed cells to both the hatching gland (hg) delimited by dots in $b$, and pharyngeal endoderm $(\mathrm{ph})(\mathrm{c})$. Each arrowhead points to a labeled cell. $(d, e)$ Fate acquired by dorsal endomesendodermal precursors under conditions of her5 overexpression. Most progenitors contributed to an increased number of pharyngeal (ph) or postpharyngeal (p.ph) endodermal cells only, whereas the hatching gland was populated only rarely. Two different embryos are shown, each arrowhead points to a labeled cell. Four other labeled cells are out of focus and thus not visible on the embryo in $d$. (whether or not overexpressing her5, see below) display a characteristic endodermal/mesendodermal behavior during gastrulation, acquiring a flattened appearance and migrating in apposition to the yolk syncitial layer, and contribute to endodermal/mesendodermal structures at $24 \mathrm{hr}$ (Fig. 6b-e). Furthermore, we confirmed (see Warga and Nüsslein-Volhard 1999) that single blastomeres of the most marginal row (row 1) of the late blastula, located within the dorsal sector (defined at the onset of gastrulation by the limits of the embryonic shield), are predominantly bipotential $(81 \%$ of cases, $n=16)$ and give rise to both hatching gland (Fig. 6b) and pharyngeal endoderm (Fig. 6c) cells. Unipotential progenitors, contributing to the hatching gland, pharyngeal endoderm, or gut only, were hit with a much lower frequency $19 \%$ of cases) (in our hands, forerunner cells were very rarely represented). We measured that single bipotential progenitors divided and specified to produce on average 5.5 hatching gland cells and 1.9 pharyngeal endoderm cells per embryo $(n=16)$. To directly test whether her5 could alter these specification choices, we performed similar fate mapping studies after overexpressing her5 at the blastoderm margin. Under these conditions, single dorsal marginal blastomeres of row 1 only rarely gave rise to hatching gland cells, but rather gave rise almost exclusively to an increased number of pharyngeal or postpharyngeal endoderm cells. On average, single progenitors divided and specified to produce 0.4 hatching gland cell and 4.2 pharyngeal or postpharyngeal endodermal cells per embryo $(n=15)$ (Fig. 6d,e). No cell death was observed. These results strongly suggest that her5 expression biases specification choices within daughter cells of endodermal/mesendodermal progenitors, such that cell contribution to the anteriormost mesendoderm is diminished in favor of an increased contribution to the intermediate endoderm.

her5 acts cell-autonomously on precursors of the anteriormost mesendoderm

Hairy/E(spl) factors generally act cell-autonomously in the regulation of cell specification (Fischer and Caudy 1998). However, Hairy was also reported to act outside its apparent expression domains during embryonic segmental patterning (Lardelli and Ish-Horowicz 1993). To determine whether her5 effects on anterior and posterior mesendodermal precursors were autonomous, or required cell interactions, we tested whether her5-expressing endodermal and mesendodermal cells could influence the specification or fate of neighboring, non-her5expressing endodermal and mesendodermal cells. To this aim we took advantage of the fact that Tar* cells, when grafted into the margin of the blastula of uninjected embryos, also selectively populate the endoderm and endmost mesendoderm (David, Sawyer, and Rosa, in prep.). We grafted small groups of Tar*-expressing cells (green) at the blastula stage within a marginal patch of (red) Tar* ${ }^{*}$ her5- or, for controls, Tar* -expressing cells (Fig. 7a). In both cases, we confirmed that Tar $^{\star}$ grafted cells contributed exclusively to endodermal and end- 

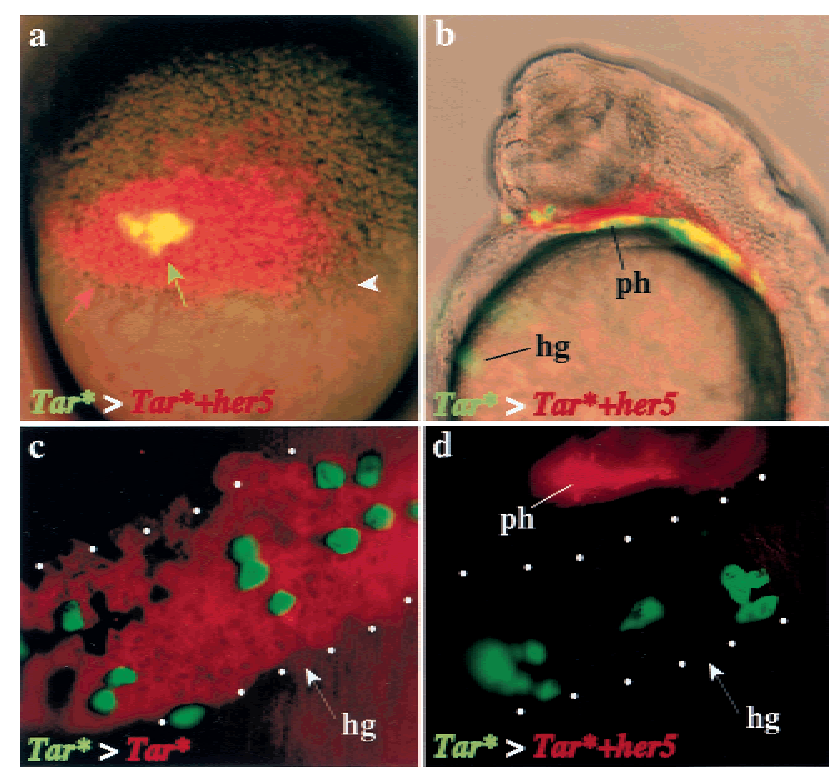

Figure 7. her5 acts cell autonomously on the anteriormost mesendoderm. (a) Grafting strategy. $\mathrm{Tar}^{\star}+\mathrm{GFP}$-expressing cells (green arrow) were grafted at the sphere stage within host embryos expressing either $\operatorname{Tar}^{\star}(c)$ or $\operatorname{Tar}^{\star}+$ her5 $(a, b, d)$ (also labeled with $2000 S$ rhodamine dextran, red arrow) at the blastoderm margin (white arrowhead). (b-d) Host embryos at $24 \mathrm{hr}$, $c$ and $d$ are frontal fluorescence views of the hatching gland region (delimited by dots), $b$ is a side view of the embryo in $c$, with fluorescence and bright field images superimposed. Independent of the nature of the host patch, the grafted cells (green) participate equally well to the host hatching gland at $24 \mathrm{hr}$ (cf. $c$ and $d$ ). Tar $^{\star}+$ her5-positive host cells (red in b,d) do not populate the hatching gland (cf. $c$ and $d$ ) but participate normally to the pharynx $(b, d)$. The superposition of green with red cells appears yellow in $a, b$. (hg) hatching gland; (ph) pharynx.

most mesendodermal structures (Fig. 7b-d). In agreement with our previous findings, we verified that Tar $^{*}+$ her5-expressing cells from the host embryo never populated the hatching gland, while they normally populated intermediate endodermal derivatives such as the pharynx (red in Fig. 7b,d). In contrast, we observed that the Tar*-positive grafted cells (green) populated the hatching gland in similar proportions regardless of the host environment, i.e., whether or not adjacent cells expressed her5 (14.4 green hatching gland cells per embryo in $\operatorname{Tar}^{\star}>$ Tar $^{\star}$ grafts, $n=13$, and 12.9 cells per embryo in Tar $^{\star}>$ Tar $^{\star}+$ her5 grafts, $n=14$ ) (Fig. 7, cf. c and d). Thus, her5 expression inhibits the development of hatching gland fate in a cell-autonomous fashion. Together with the fact that her5 acts as an active inhibitor of transcription, these results strongly suggest that her5 directly downregulates the expression of genes instrumental in imparting this anterior mesendodermal identity.

\section{her5 expression is controlled by extracellular} interactions and a Delta signaling pathway

Our results place her5 as one of the ultimate factors of a regulatory cascade imparting anterior and posterior mesendodermal specification. Therefore, it is crucial to understand the processes that control its spatio-temporal expression. As shown above, her5 is activated downstream of nodal-related signals. We wished to determine whether further additional cell interactions were required for its expression. We tested whether her5 expression could be induced in dissociated $\mathrm{Tar}^{\star}$-expressing cells. Tar* RNA injections reproducibly induced overexpression of her5 in whole embryos at the shield stage, as revealed by whole-mount in situ hybridization (data not shown) or semi-quantitative RT-PCR (Fig.8a). This induction was lost when the cells of the injected embryos were dissociated shortly after Tar $^{*}$ RNA injection and cultured until the shield stage (Fig. 8a). In contrast, the induction of $g s c$ expression by Tar* proved not sensitive to cell dissociation, thus validating our approach and the specificity of the her5 response. Therefore, the induction/maintenance of her5 expression by $\mathrm{Tar}^{*} /$ nodal signaling requires a non-cell autonomous event, and strongly suggests that a positive cell interaction process is required in vivo following activation of nodal signaling to permit the endodermal expression of her5.

One obvious candidate for the induction of her5 on cell interactions is the activation of the vertebrate Notch cascade upon Serrate/Delta binding. Consistent with this idea, both Notch- and Delta-related genes are expressed at the onset of gastrulation in zebrafish embryos (Bierkamp and Campos-Ortega 1993; Dornseifer et al. 1997; Westin and Lardelli 1997; Haddon et al. 1998). We therefore tested whether her5 expression in the endoderm could be induced by activation of Delta-Notch signaling. Quite surprisingly, injections of RNA encoding the constitutively active form of Notch, XotchDE (Haddon et al. 1998), did not lead to ectopic her5 inductions (95\% of cases, $n=44$ ), but instead diminished or abolished the endogenous expression of her 5 when they overlapped with the normal her5-positive domain (Fig. 7b). We then tested whether inhibition of the Notch-signaling cascade might have the opposite effect. Indeed, her5 expression was reproducibly induced $(100 \%$ of cases, $n=32$ ) by the truncated form of Delta, XDelta ${ }^{\text {Stu }}$ (Haddon et al. 1998) (Fig. 8d,e). XDelta ${ }^{\text {Stu }}$ lacks most of the intracellular domain of XDelta, and has a dominantnegative effect that renders its expressing cells insensitive to Delta-Notch signaling (Haddon et al. 1998). Following Delta ${ }^{\text {Stu }}$ RNA injections in a marginal blastomere at the 16-cell stage, her5 inductions were observed in deep XDelta ${ }^{\text {Stu }}$-expressing cells located at the blastoderm margin, mimicking the endogenous endodermal expression of her5 (Fig. 8d). Markers of the meso- or mesendodermal cell populations, such as $n t l, f 1 h$, and gsc, remained unaffected by XDelta ${ }^{\text {Stu }}$ injections, suggesting that XDelta ${ }^{\text {Stu }}$ expression affects a subset of these genes only (data not shown). In addition, injections of XDelta ${ }^{\text {Stu }}$ RNA proved unable to induce her5 expression in a Nodal-deficient context such as in MZoep embryos (Gritsman et al. 1999; data not shown), suggesting that Nodal signaling is required for the effect of $X$ Delta $^{\text {Stu }}$. We conclude from these experiments that, in the presence of Nodal signaling, her5 expression is in- 
a
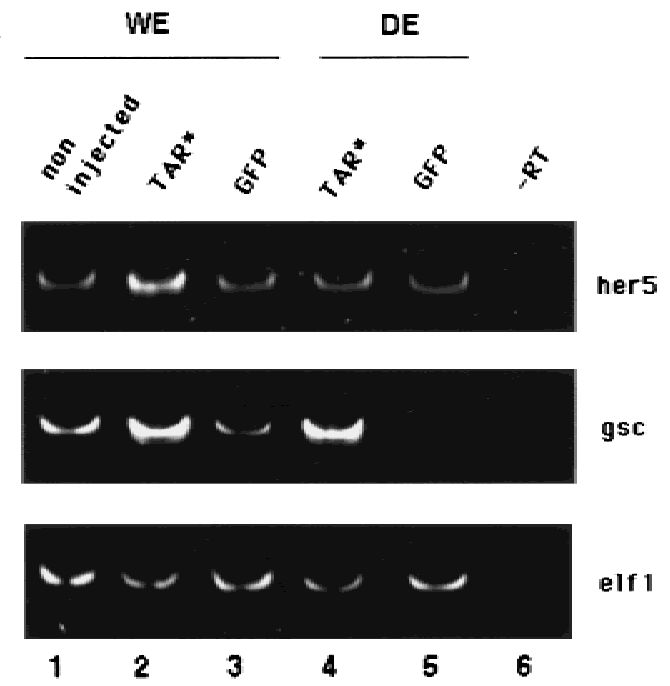

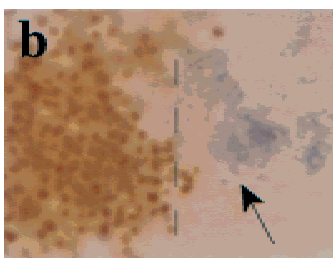

XotchDE

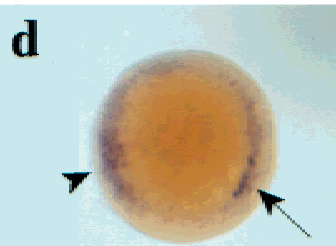

XDeltaStu

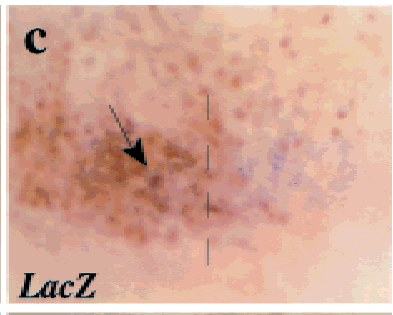

e
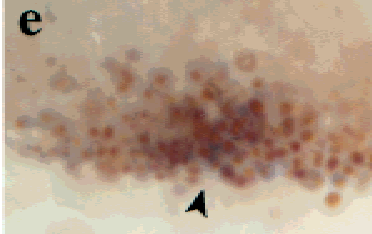

XDeltaStu
Figure 8. her5 expression requires positive cell interactions and an inhibition of Notch/Delta signaling. (a) The induction of her5 by Tar* requires positive cell interactions. RNA was extracted from whole embryos (WE, 1-3) or dissociated embryos (DE, 4-5) injected with 1 pg of Tar $^{*}$ RNA $(2,4)$ or 100 pg of GFP RNA $(3,5)$, and subsequently processed for RT-PCR. Elf1 serves as a loading control. Lanes 1 and 6 are samples from noninjected embryos treated with or without reverse transcriptase. (Endodermal) Expression of her5 is not induced upon Tar* injection when the cells of the injected embryos are dissociated; in contrast, gsc induction by Tar ${ }^{\star}$ is cell autonomous (cf. lanes 2 and 4 in each case). The basal level of her5 expression, maintained in dissociated cells (lane 5), probably corresponds to remnants of the early ubiquitous (nonendodermal) phase of her5 expression (see text). (b-e) Embryos were injected at the 16-cell stage into a marginal blastomere with 2 pg of capped XotchDE +3 pg of lacZ RNAs $(b), 3$ pg of $1 a c Z$ RNA $(c)$, or 2 pg of XDelta ${ }^{S t u}+3$ pg of lacZ RNAs $(d, e)$, and tested for her5 (in situ hybridization, blue) and betagalactosidase (immunocytochemistry, brown nuclei) (except in $d$ ) expressions at the shield stage. $(a-c, e)$ Flat-mounts of the injected areas; $(d)$ animal pole view of the embryo shown in $e$, photographed prior to betagalactosidase detection. The injected areas in $b, c$ overlapped with the endogenous axis (dorsal midline indicated by the broken line), whereas they are ventrally located in $d$,e. Endogenous her5 expression (arrows in $b-d$ ) is inhibited by XotchDE (cf. $b$ with $c$ where no inhibition is observed following expression of lac $Z$ alone). In contrast, following $\mathrm{XDelta}^{\mathrm{Stu}}$ misexpression, her5 induction (arrowheads in $d, e$ ) occurs in deep cells of the margin $(d)$, which derive from the injected blastomere (cf. blue and brown in $e$ ). hibited by the activation of the Delta/Notch cascade, and activated by the inhibition of this cascade, suggesting that a local release of Delta/Notch signaling is necessary to permit her5 expression in vivo. Taken together, our results imply that both positive and negative cell interactions are involved in the establishment of her5 expression in endodermal precursors in vivo.

\section{Discussion}

We show here that the zebrafish bHLH, Hairy/E(spl)related factor her5 is expressed in a subpopulation of dorsoanterior endodermal precursors at gastrulation, thus identifying her5 as the earliest known regional marker of the presumptive endoderm. her5 expression in this cell population requires endoderm induction and is permitted by a release of Delta/Notch signaling. At early gastrulation stages, her5 expression is progressively excluded from the presumptive territories fated to the anterior- and posteriormost mesendoderm, and her5 functions as an active transcriptional repressor to negatively control the number of cells allocated to these territories. In this process, her5 acts primarily within the endoder- mal/endmost mesendodermal lineage, and biases cell specification choices in favor of a participation to intermediate endodermal derivatives. Its negative regulation of cell contribution to the anteriormost mesendoderm is cell-autonomous. Finally, we demonstrate that this function is crucial for the patterning of the ventral forebrain and eye field. Taken together, our results shed important light on the mechanisms of vertebrate endoderm patterning, by demonstrating that a her5-mediated pathway follows endoderm/mesendoderm induction to refine cell specification in deep layers of the organizer, and jointly controls cell contribution to the anterior- and posteriormost mesendodermal domains.

\section{her5 expression and the early regionalization} of the presumptive endoderm

Our results demonstrate that her5 is markedly different from all endoderm-specific factors isolated to date: During gastrulation, her5 expression is specific of a dorsal subpopulation of endodermal precursors, and its expression does not trigger endodermal specification, but rather controls cell positional identity within the endo- 
derm and endmost mesendoderm. Thus, we identified the first gene expressed in a region-specific manner within the vertebrate presumptive endoderm, and documented its pivotal role in mediating part of the endoderm/mesendoderm regionalization process.

Because of the considerable overlap between the presumptive endodermal territories at gastrulation (Warga and Nüsslein-Volhard 1999|, it is not possible to ascertain which endodermal derivatives are populated by the progeny of her5-expressing cells. At that stage, her5 expression maps to the area from which most pharyngeal cells originate (Warga and Nüsslein-Volhard 1999), suggesting that at least part of the her5-positive population might contribute to pharyngeal derivatives. Similarly, we are unable to tell whether the endodermal cells expressing her5 at the end of gastrulation are related by lineage to the early her5-positive cells. Their distribution among the anteriormost endodermal precursors, however (Strähle et al. 1993; Rodaway et al. 1999; Fig. 5E in Warga and Nüsslein-Volhard 1999), suggests that they might also participate, at least in part, to anterior endodermal derivatives such as the pharynx. In the present study, we have demonstrated that her 5 expression at gastrulation imposed exclusion from the endmost mesendodermal domains and favored participation to the intermediate endoderm. A more extensive analysis will be required to determine whether her 5 positively engages its expressing cells toward specific derivative(s) or area(s) of the intermediate endoderm.

We sought to identify the factors which might regulate her5 expression in endodermal precursors, because of the crucial role it plays in endoderm patterning. We show that her5 expression depends on both positive and negative cell interactions acting on the endoderm/mesendoderm-fated population. We ignore at present the nature of the positive signal(s), but its identification is clearly one of our major future challenges. We unexpectedly found that her5 expression, in contrast to $E$ (spl) gene expression in other systems, is inhibited by the Notch cascade and is released by its inhibition. Consistent with this idea, Notch1a is ubiquitously expressed in the hypoblast of the early gastrula (Bierkamp and Campos-Ortega 1993), and deltaC and deltaD are strongly expressed in the prospective endoderm and mesoderm of the margin (Dornseifer et al. 1997; Haddon et al. 1998). It is tempting to speculate that Notch/Delta signaling is active throughout the margin at early gastrulation and thus initially represses her5 expression in the endoderm. A local dorsal signal might be involved in inhibiting the Notch cascade and induce her5 expression in dorsal endoderm/mesendoderm.

Taken together, our results suggest that the endodermal germ layer is regionalized following a multistep process, exemplified by the mechanisms controlling her5 expression, and by her5 function. Upon endoderm induction, both positive cell interactions and a local release of Notch/Delta signaling within endoderm/mesendodermfated cells are necessary to permit the endodermal expression of her5. The region-specific expression of her 5 evidences the existence of at least a rough molecular regionalization of the endodermal germ layer from the $30 \%$ epiboly stage. During gastrulation, the regionalization of the endoderm/mesendoderm is then refined, and this refinement locally depends on the function of her5 itself, which specifically biases cell specification choices to inhibit cell contribution to the endmost-fated mesendodermal populations, while enhancing their participation to the intermediate endoderm (see below). This sequence further suggests that the maintenance of endodermal specification (Alexander and Stainier 1999) and the regionalization of the endodermal/endmost mesendodermal germ layer are temporally overlapping events.

her5 expression controls the number of cells allocated to the anterior- and posteriormost mesendodermal domains

At the onset of gastrulation, her5 expression is progressively excluded from the prechordal plate and forerunner cell territories. Correlatively, we observed that the formation of these cell populations was prevented by ectopic her5 expression, while they developed in excess or from ectopic locations when the function of her5 was inhibited. These results suggest that her5 functions to refine endo/mesendodermal prepatterning at the dorsal embryonic margin by negatively setting the borders of the presumptive territories of the anterior- and posteriormost mesendoderm. This function is strikingly reminiscent of major instances of Hairy activity in Drosophila (see Fischer and Caudy 1998). For example, during neurogenesis, Hairy functions as a direct repressor of achaete expression to negatively delimit the proneural clusters and hence the formation of achaete-dependent sensory organs.

In this and most other processes (see Fischer and Caudy 1998), Drosophila Hairy/E(spl) proteins have been demonstrated to act on cell fate decisions, by negatively determining the position and number of specific precursor cells. A similar conclusion was attained regarding the function of Hairy-related proteins during vertebrate neurogenesis (Lee 1997; Fischer and Caudy 1998, and references therein), including zebrafish her4 (Takke et al. 1999|. Importantly, our work suggests that her5 exerts a similar function in the zebrafish early gastrula. We directly demonstrate that precursors of the endmost mesendoderm and endoderm remain within this germ layer upon overexpression of her5 (Fig. 5b and 6d,e), but that their contribution to the first territory is shifted in favor of a participation to the other: The number of hatching gland cells produced per progenitor is strongly reduced, whereas the number of pharyngeal endodermal cells is increased correspondingly (Fig. 6). Furthermore, no cell death was observed, suggesting that most hatching gland precursors were rerouted toward a pharyngeal fate rather than eliminated (data not shown). Together with the known functions of her5-related Drosophila and vertebrate factors, we believe that these observations are strong arguments to support a role of her5 in controlling decisions of cell specification, here between endodermal and endmost-mesendodermal precursors. 
Two possible restrictions must be kept in mind, however. First, we are dealing with populations of migrating cells. Formally, her5 might primarily regulate cell migration rather than cell specification, especially because its principal targets are cells that migrate the furthest during gastrulation. This hypothesis seems unlikely though, because upon perturbations of her5 expression, molecular markers identifying these populations are altered before the onset of overt cell migration (Warga and Kimmel 1990). Secondly, while our results point strongly to a direct cell specification shift from the anteriormost mesendoderm towards the intermediate endoderm upon her5 expression, they do not directly demonstrate that the reverse shift follows the forced expression of dominant-negative forms of her5 because we did not locate the precursors of the additional endmost mesendodermal cells induced by $\Delta$ basic-her5 and her5VP16. Nevertheless, $\Delta$ basic-her5 and her5-VP16 clearly trigger phenotypes opposite to those of her5 in terms of molecular markers (Fig. 2) and/or of the production of endmost mesendodermal cell types (Fig. $3 \mathrm{~m}, \mathrm{n}$ ), and coinjections demonstrate that they act by antagonizing her5 function. These results suggest that $\Delta$ basic-her5 and her5-VP16 also mediate cell specification changes, in a manner opposite to her5.

In summary, our results are best interpreted by postulating that her5 expression sets the borders of the endmost mesendodermal territories at gastrulation by regulating cell specification choices within the deep layers of the zebrafish organizer, to favor an endodermal fate at the expense of a participation to the endmost mesendoderm. Recently, a network involving Notch/Delta signaling, thus probably bHLH factors, has been proposed to assign notochord, hypochord, or floor plate cell fates within the late gastrula organizer (Appel et al. 1999). Most interestingly, our results suggest that a similar network may operate in deep regions of the early organizer to refine cell fate choices within the presumptive endoderm and endmost mesendoderm.

\section{her5 might act as a direct negative regulator of genes imparting anteriormost- and posteriormost- mesendodermal identity}

At the molecular level, we showed that her5 acts in this process as an active inhibitor of transcription (see Barolo and Levine 1997), and requires the binding of (probably Groucho-like) cofactors on its carboxy-terminal WRPW motif. In the normal embryo, the known zebrafish Groucho factors are expressed appropriately (Wülbeck and Campos-Ortega 1997) to interact with her5 and potentiate its activity. Furthermore, the dominant-negative effect of $\Delta$ basic-her5 suggests that her5 acts as a dimer. Altogether, this mode of action is reminiscent of most instances of Hairy/E(spl) activities in Drosophila (Fischer and Caudy 1998, and references therein), but in particular is in striking contrast with the activities of zebrafish her1 and her4. Interaction of the latter two proteins with Groucho factors was shown to be dispensable, and the expression of $\Delta$ basic-her 4 mutants did not inter- fere with the function of endogenous her4 (Takke and Campos-Ortega 1999; Takke et al. 1999).

Identifying the molecular targets of her5 remains an important issue. Misexpression of her 5 inhibits forerunner cells markers, including sqt (this report), and sqt mutants lack a hatching gland (Feldman et al. 1998) and are variably defective in the formation of forerunner cells, revealed by the expression of sox17 (Alexander and Stainier 1999|. However, we believe that the phenotype triggered by her5 misexpressions is not simply a secondary consequence of the perturbation of sqt expression. It was also shown that $\operatorname{Tar}^{\star}$ rescues the oep phenotype (Peyrieras et al. 1998), and thus the Nodal pathway downstream of sqt (Gritsman et al. 1999), while we demonstrate here that her5+Tar* injections reproduce her5induced defects (Fig. 5b). Thus, her5 must act downstream of sqt activity and control locally the formation of the endmost mesendoderm, i.e., by a mechanism other than a simple regulatory loop affecting sqt. The function of her5 as an active transcriptional inhibitor, together with its cell-autonomous effect on hatching gland-fated cells, strongly suggests that her5 acts by directly inhibiting genes involved in imparting this anteriormost mesendodermal identity. Among such candidate targets is the gSc gene itself, whose expression was suggested to convey anterior cell fate and/or migration in both Xenopus and chick (Cho et al. 1991; IspizuaBelmonte et al. 1993). Confirmation of this hypothesis awaits the direct molecular demonstration of her5 binding to the gSc promoter.

\section{Materials and methods}

Embryos

Embryos were obtained from natural spawning of wild-type (AB) adults, raised according to Kimmel et al. (1995).

\section{Constructs}

All her5 constructs (Fig. 2a) were subcloned into pXT7. Wildtype her5 comprises the full-length coding domain of her5 (Müller et al. 1996). Dbasic-her5 was constructed from wildtype her 5 by fusing the start methionine to the first amino acid of the HLH domain, thus removing the entire basic domain (RRVPKPLMEKRRR). $\triangle W R P W$-her5 was obtained by deleting the six carboxy-terminal amino acid-encoding region of her5 (PVWRPW). In her5-VP16, two-tandem copies of the transcriptional activator element of VP16 (DALDDFDLDML) (Seipel et al. 1994) were fused in frame to $\triangle W R P W-$ her5. To construct $\triangle O R F-h e r 5$, the entire her5 ORF was cloned into the vector pCS3+MT downstream of a myc-tag. A frameshift mutation was introduced into this construct by cloning an 11 nucleotide linker between myc and the her5 start codon. Capped mRNAs were synthesized and verified by in vitro translation.

\section{$m R N A$ injections}

Injections were carried out during the first cell cycle (lnl), or into one marginal blastomere of the 16-celled embryo (100pl), together with the lineage tracers 2000S rhodamin-dextran, 2000S fluorescein-dextran, or GFP mRNA (50 pg), and only em- 
bryos having received the injection at the appropriate location along the margin (sorted out at the shield stage under fluorescence) were analyzed. All mRNAs were coinjected with lacZ 16 $\mathrm{pg})$ or GFP (5 pg) mRNAs and the distribution of the injected progeny was verified a posteriori by anti-betagalactosidase or anti-GFP immunocytochemistry (see below), or under fluorescence.

Fate mapping of endodermal/mesendodermal progenitors under conditions of sustained her5 expression

A solution of 10-kD DMNB-caged fluorescein $(5 \mathrm{mg} / \mathrm{ml})$ (Mo-

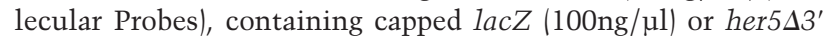
(20 ng/ $\mu \mathrm{l})$ RNA, was injected into one marginal blastomere at the 16 -cell stage. When embryos reached the $40 \%$ epiboly stage, the dye was activated in a single blastomere of the most marginal row by a microlaser beam, as described in Serbedjiza et al. (1988). At the shield stage, the location of the labeled blastomere was assessed and only embryos labeled in the dorsal sector defined by the borders of the embryonic shield were kept. The behavior and location of the labeled cell(s) were analyzed during gastrulation and at $24 \mathrm{hr}$ by visual inspection.

\section{Cell dissociation experiments and semi-quantitative RT-PCR}

Cells from pools of 10 injected embryos were dissociated at the sphere stage in calcium-free Ringer medium, and cultured at $28.5^{\circ} \mathrm{C}$ for two hours, or until control embryos reached the shield stage. Total RNA was extracted from dissociated cells or whole embryos using the TRIzol reagent (GIBCO BRL), and $1 \mu \mathrm{g}$ of RNA was processed for reverse transcription with the MMLV-transcriptase (Stratagene). 1/50 of the reaction was subsequently used for PCR. All PCR amplifications were carried out with an annealing temperature of $56^{\circ} \mathrm{C}$. The number of cycles required to be in the exponential range was determined for each pair of primers in preliminary test experiments. RTPCR products were resolved on a $6 \%$ nondenaturing polyacrylamide gel, subsequently stained with SYBR Green. The primers used were as follows (number of cycles in brackets). her5 (26): upstream: TAGTAGACCTAGCTGGTCTTTTCAGTCTTTGGAGAGC; downstream: TAAAAAGGGCACAGCACAGAGGAGAGTGATGAGGATGT; gsc (26): upstream: GGGATGTTTAGTATCGACAG; downstream: GCTGTCAGAACCACGTCGCT; Elf1 (20): upstream: TCACCCTGGGAGTGAAACAGC; downstream: ACTTGCAGGCGATGTGAGCAG.

\section{Grafting experiments}

Donor embryos were injected at the 4-cell stage into one blastomere with $0.06 \mathrm{pg}$ of $\mathrm{Tar}^{*}+5 \mathrm{pg}$ of GFP mRNAs. Host embryos were injected at the 16-cell stage into a marginal blastomere with rhodamine dextran $+0.06 \mathrm{pg}$ of $\mathrm{Tar}^{*}+5 \mathrm{pg}$ of $1 \mathrm{ca} Z$ mRNAs \pm 3 pg of her 5 mRNA. At the sphere stage, 5-10 donor (green) cells were grafted in the center of the recipient (red) patch. Grafted embryos were observed at $24 \mathrm{hr}$.

\section{Phenotypic analyses}

In situ hybridization (ISH) and immunocytochemistry were done following standard protocols (Hauptmann and Gerster 1994). For the immunodetection of GFP, the mAb 3E6 (Quantum Biotechnologies) was used at $1 / 1000$. Sectioning of wholemount ISH-stained embryos was performed with cryostat (10 $\mu \mathrm{m}$ sections, Fig. 1g,k,m,n) or JB4 resin embedding (Polysciences) and ultramicrotomy (Fig. 1h-j).

\section{Acknowledgments}

We thank Drs. J.A. Campos-Ortega, M. Rebagliati, I. Dawid, and M. Volovitch for reagents, and L. Saint-Etienne for technical assistance. We are grateful to M. Brand, G. Guimera, F. Schweisguth, and A.Vincent for critical reading of the manuscript, B. Appel and J. Eisen for communicating results prior to publication, and S. Bray and K. Lawson for helpful discussions. Financial support was provided by the EC and HFSP to W.W. and M.W., and by the Association pour la Recherche contre le Cancer, Ligue Nationale contre le Cancer, and Ministére de la Recherche to F.R.

The publication costs of this article were defrayed in part by payment of page charges. This article must therefore be hereby marked "advertisement" in accordance with 18 USC section 1734 solely to indicate this fact.

\section{References}

Alexander, J. and Stainier, D.Y.R. 1999. A molecular pathway leading to endoderm formation in zebrafish. Curr. Biol. 9: $1147-1157$.

Appel, B., Fritz, A., Westerfield, M., Grunwald, D.J., Eisen, J.S., and Riley, B.R. 1999. Delta-mediated specification of midline cell fates in zebrafish embryos. Curr. Biol. 9: 247-256.

Barolo, S. and Levine, M. 1997. Hairy mediates dominant repression in the Drosophila embryo. EMBO J. 16: 28832891.

Bierkamp, C. and Campos-Ortega, J.A. 1993. A zebrafish homologue of the Drosophila neurogenic gene Notch and its pattern of transcription during early embryogenesis. Mech. Dev. 43: $87-100$.

Cho, K.W., Blumberg, B., Steinbeisser, H., and de Robertis, E.M. 1991. Molecular nature of Spemann's organizer: The role of the Xenopus homeobox gene goosecoid. Cell 20: 11111120 .

Cooper, M.S. and D'Amico, L.A. 1996. A cluster of noninvoluting endocytic cells at the margin of the zebrafish blastoderm marks the site of embryonic shield formation. Dev. Biol. 180: $184-198$.

Dawson, S.R., Turner, D.L., Weintraub, H., and Parkhurst, S.M. 1995. Specificity for the hairy/Enhancer of split basic helixloop-helix (bHLH) proteins maps outside the bHLH domain and suggests two separable modes of transcriptional repression. Mol. Cell. Biol. 15: 6923-6931.

Dornseifer, P., Takke, C., and Campos-Ortega, J.A. 1997. Overexpression of a zebrafish homologue of the Drosophila neurogenic gene Delta perturbs differentiation of primary neurons and somite development. Mech. Dev. 63: 159-171.

Ermakova, G.V., Alexandrova, E.M., Kazanskaya, O.V., Vasiliev, O.L., Smith, M.W., and Zaraisky, A.G. 1999. The homeobox gene, Xanf-1, can control both neural differentiation and patterning in the presumptive anterior neurectoderm of the Xenopus laevis embryo. Development 126: 4513-4523.

Feldman, B., Gates, M.A., Egan, E.S., Dougan, S.T., Rennebeck, G., Sirotkin, H.I., Schier, A.F., and Talbot, W.S. 1998. Zebrafish organizer development and germ-layer formation require nodal-related signals. Nature 395: 181-185.

Fisher, A. and Caudy, M. 1998. The function of hairy-related bHLH repressor proteins in cell fate decisions. BioEssays 20: 298-306.

Gritsman, K., Zhang, J., Cheng, S., Heckscher, E., Talbot, W.S., and Schier, A.F. 1999. The EGF-CFC protein One-Eyed Pinhead is essential for Nodal signaling. Cell 97: 121-132.

Haddon, C., Smithers, L., Schneider-Maunoury, S., Coche, T., 
Henrique, D., and Lewis, J. 1998. Multiple delta genes and lateral inhibition in zebrafish primary neurogenesis. Development 125: 359-370.

Hauptmann, G. and Gerster, T.C. 1994. Two-colour wholemount in situ hybridization to vertebrate and Drosophila embryos. Trends Genet. 10: 266.

Henry, G.L., Brivanlou, I.H., Kessler, D.S., Hemmati-Brivanlou, A., and Melton, D.A. 1996. TGF- $\beta$ signals and a prepattern in Xenopus laevis endodermal development. Development 122: 1007-1015.

Ispizua-Belmonte, J.C., de Robertis, E.M., Storey, K.G., and Stern, C.D. 1993. The homeobox gene goosecoid and the origin of organizer cells in the early chick blastoderm. Cell 74: 645-659.

Jimenez, G., Pinchin, S.M., and Ish-Horowicz, D. 1996. In vitro interactions of the Drosophila Hairy and Runt transcriptional repressors with target promoters. EMBO J. 15: 70887098.

Joseph, E. and Melton, D.A. 1998. Mutant Vg1 ligands disrupt endoderm and mesoderm formation in Xenopus embryos. Development 125: 2677-2685.

Kimmel, C.B., Ballard, W.W., Kimmel, S.R., Ullmann, B., and Schilling, T.F. 1995. Stages of embryonic development of the zebrafish. Dev. Dyn. 203: 253-310.

Lee, J.E. 1997. Basic helix-loop-helix genes in neural development. Curr. Op. Neurobiol. 7: 13-20.

Melby, A.E., Warga, R.M., and Kimmel, C.B. 1996. Specification of cell fates at the dorsal margin of the zebrafish gastrula. Development 122: 2225-2237.

Müller, M., v. Weizsäcker, E., and Campos-Ortega, J.A. 1996. Transcription of a zebrafish gene of the hairy-Enhancer of split family delineates the midbrain anlage in the neural plate. Dev. Genes Evol. 206: 153-160.

Palmeirim, I., Henrique, D., Ish-Horowicz, D., and Pourquié, O. 1997. Avian hairy gene expression identifies a molecular clock linked to vertebrate segmentation and somitogenesis. Cell 91: 639-648.

Pera, E.M. and Kessel, M. 1997. Patterning of the chick forebrain anlage by the prechordal plate. Development 124:41534162.

Peyrieras, N., Strähle, U., and Rosa, F. 1998. Conversion of zebrafish blastomeres to an endodermal fate by TGF-b-related signalling. Curr. Biol. 8: 783-786.

Rebagliati, M.R., Toyama, R., Fricke, C., Haffter, P., and Dawid, I.B. 1998. Zebrafish nodal-related genes are implicated in axial patterning and establishing left-right asymmetry. Dev. Biol. 199: 261-272.

Rodaway, A., Takeda, H., Koshida, S., Broadbent, J., Price, B., Smith, J.C., Patient, R., and Holder, N. 1999. Induction of the mesendoderm in the zebrafish germ ring by yolk cellderived TGF-beta family signals and discrimination of mesoderm and endoderm by FGF. Development 126:30673078 .

Sampath, K., Rubinstein, A.L., Cheng, A.M.S., Liang, J.O., Fekany, K., Solnica-Krezel, L., Korzh, V., Halpern, M.E., and Wright, C.V.E. 1998. Induction of the zebrafish ventral brain and floorplate requires cyclops/nodal signalling. Nature 395: 185-189.

Schier, A.F. and Shen, M.M. 2000. Nodal signaling in vertebrate development. Nature 403: 385-389.

Schneider, V.A. and Mercola, M. 1999. Spatially distinct head and heart inducers within the Xenopus organizer region. Curr. Biol. 9: 800-809.

Schulte-Merker, S., Ho, R.K., Hermann, B.G., and Nüsslein-Volhard, C. 1992. The protein product of the zebrafish homologue of the mouse $T$ gene is expressed in nuclei of the germ ring and the notochord of the early embryo. Development 116: $1021-1032$.

Schulte-Merker, S., Hammerschmidt, M., Beuchle, D., Cho, K.W., De Robertis, E.M., and Nüsslein-Volhard, C. 1994. Expression of zebrafish goosecoid and no tail gene products in wild-type and mutant no tail embryos. Development 120: $843-852$.

Seipel, K., Georgiev, O., and Schaffner, W. 1994. A minimal transcription activation domain consisting of a specific array of aspartic acid and leucine residues. Biol. Chem. HoppeSeyler 375: 463-470.

Serbedzija, G.N., Chen, J-N., and Fishman, M.C. 1988. Regulation in the heart field of zebrafish. Development 125: 10951101.

Shih, J. and Fraser, S.E. 1995. Distribution of tissue progenitors within the shield region of the zebrafish gastrula. Development 121: 2755-2765.

Stachel, S.E., Grunwald, D.J., and Myers, P.Z. 1993. Lithium perturbation and goosecoid expression identifies a dorsal specification pathway in the pregastrula zebrafish. Development 117: 1261-1274.

Strähle, U., Blader, P., Henrique, D., and Ingham, P.W. 1993. Axial, a zebrafish gene expressed along the developing body axis, shows altered expression in cyclops mutant embryos. Genes \& Dev. 7: 1436-1446.

Takke, C. and Campos-Ortega, J.A. 1999. her1, a zebrafish pairrule like gene, acts downstream of notch signalling to control somite development. Development 126: 3005-3014.

Takke, C., Dornseifer, P., v. Weisäcker, E., and Campos-Ortega, J.A. 1999. her4, a zebrafish homologue of the Drosophila neurogenic gene $E(s p 1)$, is a target of Notch signalling. Development 126: 1811-1821.

Thisse, C., Thisse, B., Halpern, M.E., and Postlethwait, J.H. 1994. Goosecoid expression in neuroectoderm and mesendoderm is disrupted in zebrafish cyclops gastrulas. Dev. Biol. 164: 420-429.

Warga, R.M. and Kimmel, C.B. 1990. Cell movements during epiboly and gastrulation in zebrafish. Development 108: 569-580.

Warga, R.M. and Nüsslein-Volhard, C. 1999. Origin and development of the zebrafish endoderm. Development 126: 827838.

Weiszäcker, E.v. 1994. "Molekulargenetische Untersuchungen an sechs Zebrafisch-Genen mit Homologie zur Enhancer of split Gen-Familie von Drosophila." Inaugural Dissertation, Universität zu Köln.

Westin, J. and Lardelli, M. 1997. Three novel Notch genes in zebrafish: Implications for vertebrate Notch gene evolution and function. Dev. Genet. Evol. 207: 51-63.

Wülbeck, C. and Campos-Ortega, J.A. 1997. Two zebrafish homologues of the Drosophila neurogenic gene groucho and their pattern of transcription during early embryogenesis. Dev. Genes Evol. 207: 156-166.

Yasuo, H. and Lemaire, P. 1999. A two-step model for the fate determination of presumptive endodermal blastomeres in Xenopus embryos. Curr. Biol. 9: 869-879.

Zorn, A.M., Butler, K., and Gurdon, J.B. 1999. Anterior endomesoderm specification in Xenopus by Wnt/ $\beta$-catenin and TGF- $\beta$ signalling pathways. Dev. Biol. 209: 282-297.

Zuber, M.E., Perron, M., Philpott, A., Bang, A., and Harris, W.A. 1999. Giant eyes in Xenopus laevis by overexpression of Xoptx2. Cell 98: 341-52. 


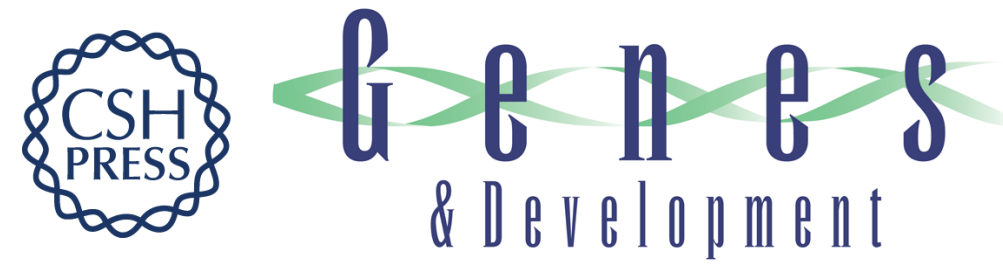

\section{Coregulation of anterior and posterior mesendodermal development by a hairy-related transcriptional repressor}

Laure Bally-Cuif, Carole Goutel, Marion Wassef, et al.

Genes Dev. 2000, 14:

Access the most recent version at doi:10.1101/gad.14.13.1664

References

This article cites 47 articles, 19 of which can be accessed free at: http://genesdev.cshlp.org/content/14/13/1664.full.html\#ref-list-1

License

Email Alerting

Receive free email alerts when new articles cite this article - sign up in the box at the top Service right corner of the article or click here.

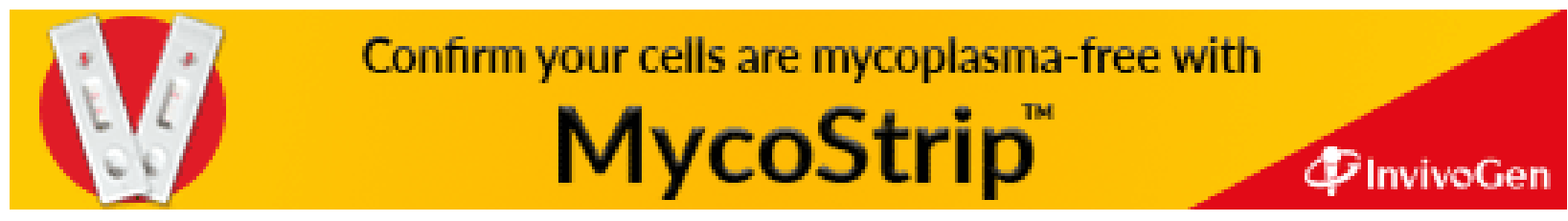

